\title{
COVID-19 e organizações: estratégias de enfrentamento para redução de impactos
}

\author{
Beatriz Leite Gustmann de Castro, ${ }^{1, *}$, Joice Beatriz Bock de Oliveira², \\ Lísia Quoos Morais ${ }^{3}$, Maria Julia Pegoraro Gai ${ }^{4}$
}

\footnotetext{
${ }^{1}$ http://orcid.org/0000-0001-8205-2979 / Universidade Federal de Santa Maria (UFSM), Brasil

2 http://orcid.org/0000-0001-8441-8399 / Universidade Federal de Santa Maria (UFSM), Brasil

${ }^{3}$ http://orcid.org/0000-0001-5649-6912 / Universidade Federal de Santa Maria (UFSM), Brasil

${ }^{4}$ http://orcid.org/0000-0002-8481-1112 / Universidade Federal de Santa Catarina (UFSC), Brasil
}

\section{Resumo}

As organizações sofrem com os impactos socioeconômicos do cenário de crise global provocados pela pandemia de COVID-19. Nesse contexto é primordial adotar medidas objetivas para minimizar os reflexos nocivos dentro da gestão organizacional. Este estudo buscou identificar as principais estratégias para redução de impactos da COVID-19 nas organizações brasileiras. Trata-se de um ensaio teórico baseado em uma revisão de literatura, através de análise de artigos de bases a partir de dados nacionais e internacionais sobre a COVID-19, bem como investigação relacionada às experiências em pandemias anteriores, assim como a legislação brasileira vigente no período pandêmico. Considerando esse cenário de incertezas, sugere-se a adoção de estratégias para minimizar impactos desfavoráveis às atividades corporativas, tais como: comunicação eficiente; planejamento do trabalho; aprimoramento digital; tele-trabalho; adoção de medidas visando o bem-estar dos trabalhadores. Identificou-se a necessidade de planejamentos emergenciais para o enfrentamento da pandemia, incluindo: políticas empáticas, fornecimento de suporte para tele-trabalho e ações para adaptação e manutenção organizacional durante a crise.

Palavras-chave: COVID-19, enfrentamento, organizações.

COVID-19 and organizations: coping strategies to reduce impacts

Abstract

Organizations have suffered from the socioeconomic impacts of the global crisis scenario caused by the COVID-19 pandemic. In this context, it is essential to adopt objective measures to minimize harmful effects on organizational management. This study sought to identify the main strategies for reducing the impacts of COVID-19 on Brazilian organizations. It is a theoretical essay based on a literature review of articles from national and international databases, covering COVID-19, experiences in previous pandemics, as well as Brazilian legislation in force during the pandemic period. Considering this scenario of uncertainties, it is suggested to adopt strategies to minimize unfavorable impacts on corporate activities, such as efficient communication, work planning, digital enhancement, teleworking, and adoption of measures aimed at the welfare of workers. The need for emergency planning to face the pandemic was identified, with measures including empathic policies, provision of support for telework, and actions for adaptation and organizational maintenance during the crisis.
COVID-19 y organizaciones: estrategias de afrontamiento para reducción de los impactos

Resumen

Las organizaciones sufren los impactos socioeconómicos del escenario de crisis global causado por la pandemia de COVID-19. En este contexto es esencial adoptar medidas objetivas para minimizar los efectos nocivos dentro de la gestión organizacional. Este estudio buscó identificar las principales estrategias para reducir los impactos de la COVID-19 en las organizaciones brasileñas. Es un ensayo teórico basado en una revisión de literatura, a través del análisis en artículos de bases a partir de datos nacionales e internacionales sobre COVID-19, bien como investigación relacionada a las experiencias en pandemias anteriores, así como la legislación brasileña vigente en el período de la pandemia. Considerando este escenario de incertidumbres, se sugiere la adopción de estrategias para minimizar los impactos desfavorables en las actividades corporativas, tales como: comunicación eficiente; planificación del trabajo; mejora digital; teletrabajo; adopción de medidas destinadas al bienestar de los trabajadores. Se identificó la necesidad de una planificación de emergencia para enfrentar la pandemia, que incluye: políticas empáticas, provisión de apoyo para el teletrabajo y acciones para la adaptación y el mantenimiento de la organización durante la crisis.

Palabras-clave: COVID-19, coping, trabajo. 
O Brasil e o mundo estão diante de uma crise sem precedentes. Os efeitos do novo coronavírus (COVID-19) estão produzindo impactos devastadores na saúde, na economia e na dinâmica do comportamento dos profissionais e das organizações. A aplicação de medidas restritivas ao deslocamento das pessoas voltadas ao isolamento social busca mitigar os efeitos da exposição ao contágio e promover o controle da pandemia em termos de atendimento às situações de emergência e uso de equipamentos para manutenção da vida (Barros-Delben et al., 2020; Cruz et al., 2020).

Os efeitos da atual crise certamente se estenderão por meses e talvez anos, comprometendo cenários socioeconômicos anteriormente previstos (Silva, 2020). Trata-se, de fato, de um choque de alta intensidade na vida das populações e na capacidade das organizações em manterem suas rotinas operacionais, metas, produtividade e expectativas. No entanto, nem todos os países sinalizam agilidade na inclusão de medidas preventivas diante da irrupção causada pela COVID-19 no sistema público de saúde (Kandel, Chungong, Omaar, \& Xing, 2020). Nesse contexto de pandemia global, tornou-se necessária a implementação de estratégias e recomendações já conhecidas em função de outras experiências para uma atuação efetiva em emergências dessa natureza (Sohrabi et al, 2020).

Nesse cenário, torna-se indispensável que o espírito colaborativo entre países, instituições e área médica seja acentuado, especialmente em relação ao compartilhamento de experiências e conhecimento para contenção do vírus (Wong, Leo, \& Tan, 2020) e preparação das pessoas e organizações para a convivência no contexto atual de instabilidade econômica e social. A adoção de estratégias tradicionais utilizadas em outras epidemias - como a SARS - e os conhecimentos decorrentes da disseminação desse vírus são importantes no planejamento das medidas contra a COVID-19, pois ainda que não sejam capazes de conter totalmente o surto da doença, poderão ser fundamentais para diminuição do pico de incidência e mortes (Wilder-Smith, Chiew, \& Lee, 2020).

As decisões governamentais devem considerar as questões culturais e populacionais (Wang, Ng, \& Brook, 2020), visando adaptar as estratégias para uma atuação incisiva. Medidas de quarentena, isolamento social e restrições à movimentação das pessoas mostraram-se, em diferentes países, como as melhores estratégias de combate à proliferação da doença, em função dos riscos de crescimento exponencial do contágio na população e da possibilidade de exaustão dos sistemas de saúde (Croda et al., 2020).

A crise proporcionada pela COVID-19 atingiu sistemicamente a mobilidade social, a gestão, o planejamento e as operações do Estado e das organizações, com impactos importantes nos empregos e na sustentabilidade econômica e social. A preocupação de empresários e trabalhadores acerca da continuidade de comercialização de produtos e/ou serviços, muitas vezes essenciais à comunidade, acelerou a adoção de práticas de gerenciamento e suporte a processos de trabalhos virtuais ou remotos. Desse modo, a ameaça e temor de não poder garantir postos de trabalho e renda em diversas organizações, as incertezas quanto à extensão das medidas adotadas até o momento e as consequências sociais e econômicas acentuam as preocupações com o cenário pós-crise. As mudanças experimentadas em função da pandemia provocaram alterações nas rotinas de trabalho e no comportamento corporativo, com revisão das reais necessidades de manutenção de processos e estruturas, do mesmo modo que encoraja as organizações a migrarem para o e-commerce e a promoverem novas estratégias de gestão.

Frente a esse cenário, este trabalho tem por objetivo identificar as principais estratégias para redução de impactos da COVID-19 nas organizações brasileiras. Para alcançar este objetivo, o método proposto é elucidado na seção a seguir.

\section{Método}

Para dar luz às indagações discorridas neste estudo, realizou-se uma revisão de literatura a partir de estudos recentes sobre os impactos da COVID-19, elencando as principais estratégias para redução dos efeitos da pandemia nas organizações brasileiras. Para isso, consultou-se as bases de dados da Web of Science, Scielo e Scopus e a legislação específica para a COVID-19 no contexto brasileiro, tal como sites governamentais que possuem informações relacionadas a pandemia e tomada de decisões acerca das organizações.

Os critérios de inclusão utilizados basearam-se na seleção de artigos a partir dos descritores “TS=(("pandemic") AND ("work" OR "organization"))", publicados entre os anos de 2011 a 2020 que apresentassem estudos acerca de experiências anteriores de pandemias ou epidemias e também estudos mais recentes específicos sobre o novo coronavírus relacionados ao trabalho e ao contexto organizacional.

\section{Resultados e Discussão}

Esta seção expõe os resultados na consecução da pesquisa, alinhando-os ao objetivo proposto. A análise dos resultados está disposta em quatro tópicos. No primeiro são abordadas as medidas adotadas pelo Governo Federal frente à pandemia voltadas às diferentes organizações. $\mathrm{O}$ segundo discorre sobre as principais alternativas que emergem como soluções para manutenção e sustentabilidade das atividades econômicas, em que se destacam as estratégias laborais e mercadológicas com uso de ambientes digitais. No terceiro, o enfoque visou a apresentação do panorama do teletrabalho. Por fim, o quarto tópico versa sobre a necessidade de atenção à saúde mental no contexto da pandemia.

\section{Medidas Governamentais Frente à Pandemia}

Diante dos avanços da COVID-19, o governo brasileiro adotou medidas no final de janeiro por meio do Decreto $\mathrm{n}^{0} 10.211$ (2020), dispondo sobre o Grupo Executivo Interministerial de Emergência em Saúde Pública de Importância Nacional e Internacional. Em 3 de fevereiro declarou-se Emergência em Saúde Pública de Importância Nacional, conforme Portaria $n^{\circ} 188$ (2020). Tardiamente, em 22 de março, o Governo Federal editou a Medida Provisória no 927 (2020), que trata de medidas trabalhistas alternativas para enfrentamento do estado de calamidade pública como adoção de antecipação de feriados e férias individuais e coletivas, utilização de banco de horas, interrupção de obrigações administrativas em saúde e segurança no trabalho, suspensão do recolhimento do FGTS, orientação para os funcionários utilizarem o momento para qualificação e uso do teletrabalho.

De forma complementar, editou-se a Medida Provisória $n^{\circ}$ 936 (2020) em 1 de abril, que induz o Programa Emergencial de Manutenção do Emprego e da Renda, permitindo redução da jornada de trabalho e de salário e suspensão temporária de contratos, além de instituir um benefício emergencial de preservação do emprego e renda. Por meio da Medida Provisória no 944 (2020) é instituído o Programa Emergencial de Suporte a Empregos, destinado à realização de operações de crédito com empresários, sociedades empresariais e sociedades cooperativas para o pagamento da folha salarial dos colaboradores. Por fim, a Resolução n ${ }^{\circ} 154$ (2020) de 3 de abril, do Comitê Gestor do Simples Nacional, prorroga o prazo para pagamento do ICMS e ISS no âmbito do Simples Nacional. O conjunto dessas medidas pode ser utilizado pelas organizações para minimizar os impactos dos prejuízos econômicos e preservação dos contratos de trabalho.

As medidas relacionadas a licença de trabalhadores apresen- 
tam resultados positivos para contenção do vírus, mas não são eficazes quando há disseminação ininterrupta na comunidade, uma vez que os mesmos trabalhadores podem ser contaminados pela exposição ao vírus (Parodi \& Liu, 2020). O rastreamento e isolamento de pessoas contaminadas ainda é fonte de controvérsia política e científica. Há dificuldade em determinar o grau de eficácia para cada política de contenção não farmacológica, que não conta com remédios ou vacinas (Hellewell et al., 2020) e, por isso, é necessário adotar medidas de disseminação de informação sobre cuidados à saúde da população e preparar os profissionais e sistemas de saúde para o excesso de demanda (Bedford et al., 2020; Gatera \& Pavarini, 2020). O auxílio financeiro emergencial desencadeado pelo Governo Federal por meio da Medida Provisória $n^{\circ} 936$ (2020) busca minimizar os problemas de subsistência dos trabalhadores autônomos. Medida similar a essa pode ser utilizada pelas organizações a fim de reduzir os problemas financeiros que se avolumam entre seus clientes internos e externos.

De maneira articulada com as estratégias disponibilizadas pelo governo, as organizações utilizam recursos para reduzir os impactos da pandemia. Entre esses recursos, destaca-se os ambientes digitais, especialmente em decorrência das medidas de isolamento social.

\section{Ambientes Digitais: Estratégias Laborais e Mercadológicas}

Ambientes virtuais, de acesso sempre ativo, a qualquer hora, em qualquer lugar e de variados meios tecnológicos promovem a interação em redes cada vez mais intensas nas organizações (Chui \& Chan, 2019). A evolução das tecnologias de informação e de comunicação (TIC) promoveu mudanças importantes na sociedade ao impulsionar o acesso direto das pessoas à informação e ao propiciar maior interação entre grupos de interesses no meio virtual, no qual tem migrado elementos relacionados ao consumo e à economia, introduzindo novos padrões de mercado e de organização produtiva das organizações (Chaves Júnior, 2017; Felstiner, 2011; Wu, Liang, \& Zhang, 2020).

Nessa situação de crise, uma das formas de potencializar as vendas e minimizar perdas financeiras é investir em marketing por meio de mídias digitais. Com essa estratégia, os e-business utilizam-se da análise de dados para publicizar e manter suas transações mercadológicas ativas (Behera, Gunasekaran, Gupta, Kamboj, \& Bala, 2020). Em um sentido mais amplo, as TICs intensificam relacionamentos entre usuários, clientes, organizações e colaboradores (Rindfleisch, 2019).

As TICs têm sido potencializadas no período de quarentena, especialmente em home office e no gerenciamento de negócios digitais, estratégias que começam a receber mais atenção por parte das organizações (Huang et al., 2020). A crise de saúde global causada pela COVID-19 gerou novos desafios à gestão de plataformas digitais eficientes, estáveis e acessíveis para um grande número de pessoas em uma quantidade limitada de tempo e recursos (Huang et al., 2020; Yaseen \& Al Omoush, 2020). Dessa maneira, com a crescente utilização do espaço virtual como forma de trabalho, o teletrabalho configura-se como uma das principais estratégias para continuação das atividades laborais.

\section{O Teletrabalho como Alternativa}

O termo teletrabalho está associado ao trabalho realizado remotamente, por meio da tecnologia da informação, possibilitando a obtenção dos resultados do trabalho em um local diferente daquele ocupado pelo que o realiza (Messenger, 2019). Essa modalidade tende a auxiliar na redução de custos, aumento da produtividade e aproveitamento do tempo para a demanda de atividades. Com isso, o teletrabalho é uma opção para al- cançar objetivos organizacionais, ainda que haja desafios para implementação, como as adversidades de infraestrutura tecnológica, dificuldade de adaptação, perda de vínculo com organização, dificuldade de comunicação, incerteza sobre a avaliação e falta de reconhecimento (Filardi, Castro, \& Zanini, 2020).

Com o intuito de reduzir gastos e maximizar os resultados, o teletrabalho tende a ser uma opção, porém há resistências na implantação devido às adversidades apresentadas. Além de mitigar as despesas acerca da estrutura ou manutenção, essa configuração laboral garante maior qualidade de vida aos colaboradores pela flexibilidade, convívio com a família e por não ter perda de tempo no trajeto até o trabalho (Veiga, Oliveira, \& Mendes, 2020). Decorrente da rápida disseminação do novo coronavírus, as organizações não possuíam planos de contingência e, neste sentido, Alves e Lopes (2019) apontam a relevância de uma comunicação efetiva entre líderes e equipes para responder e transmitir as informações necessárias aos demais colaboradores.

A modalidade de teletrabalho tem sido utilizada pelas equipes médicas para o combate ao coronavírus, desde o processo de triagem dos pacientes até as consultas clínicas de modo virtual. Ainda que seja necessária a implementação de uma estrutura, principalmente em hospitais, a telemedicina se revela uma medida eficaz, especialmente para orientação de medidas preventivas (Carr \& Hollander, 2020). O teletrabalho denota desafios para sua implementação e gestão, uma vez que, a modalidade não é adequada para todas as circunstâncias (Messenger, 2019). Ademais, esta modalidade de trabalho demanda atentar para aspectos relacionados à saúde mental, já que favorece a uma ruptura de contato físico com outras pessoas a qual os colaboradores estavam habituados.

\section{Atenção à Saúde Mental}

A situação gerada pelo novo coronavírus tem gerado alterações de humor e dificuldade de reação aos sintomas de estresse, aspectos que contribuem para a instabilidade das organizações. Encontrar estratégias que minimizem os impactos negativos da saúde mental das pessoas deve estar no centro das políticas organizacionais. $\mathrm{O}$ temor pela contaminação e os efeitos sociais e econômicos da quarentena atingem significativamente a saúde mental de um grande contingente de pessoas (Brooks et al., 2020).

As TICs podem ser uma importante ferramenta no monitoramento da saúde pública, assim como na disseminação de medidas de prevenção e tratamento da COVID-19 e seus efeitos na saúde mental (Ting, Carin, Dzau, \& Wong, 2020). Assim, a comunicação corporativa também pode ser utilizada como estratégia de cuidado com a saúde mental dos funcionários e no enfrentamento das consequências da paralisação das organizações em função da COVID-19 (Asmundson \& Taylor, 2020), podendo ser uma importante ferramenta gerencial de crises durante e após a pandemia, mantendo um canal de diálogo com os colaboradores (Duan \& Zhu, 2020).

A ansiedade, frustração e medo afetam os profissionais das mais diversas áreas em meio a pandemia. Os profissionais que atuam na linha de frente ao combate à COVID-19, seja nos hospitais, nos centros de abastecimento ou nas comissões de gestão direta da crise são, de fato, os mais expostos à contaminação, assim como seus familiares. Serviços de atendimento psicológico por meios de TICs podem ser uma estratégia importante de promoção da saúde mental (Chen et al., 2020; Wong et al., 2020).

Aos que estão em casa, em férias individuais ou coletivas, por exemplo, a ansiedade se apresenta pelo medo de não ter um emprego para o qual voltar (Filardi et al., 2020). Em situações como essa, a educação em saúde pública e a veiculação de informações estritamente científicas podem conter a ansiedade da população como um todo e auxiliar ativamente no combate à pandemia por meio das me- 
didas preventivas (Xiao \& Torok, 2020). Medidas de cuidados com a saúde mental dos colaboradores devem ser planejadas, inclusive para o período pós-pandemia, pelo risco de desenvolvimento de estresse pós-traumático (American Psychiatric Association, 2014).

Aos que estão no regime de home office torna-se ansiogênico esforçar-se todos os dias para manter uma rotina de produção dentro dos horários e padrões praticados no trabalho presencial. É essencial que haja um gerenciamento das expectativas em torno dessa nova modalidade, considerando que as condições de trabalho não serão as mesmas, logo, os resultados também não serão. Por isso, é notável estabelecer objetivos mensuráveis e não esperar plena produção desde o primeiro dia, visto que a adaptação leva tempo (Aderaldo, Aderaldo, \& Lima, 2017).

\section{Considerações Finais}

Não há respostas certas ou estratégias simples frente a cenários incertos, sobretudo quando esses cenários se configuram crises globais. A exemplo da China, primeiro país a conviver com a COVID-19, que participa de quase todos os setores da economia global, experimenta a mais baixa taxa de crescimento em 30 anos. As províncias responsáveis por mais de $90 \%$ das exportações chinesas ordenaram, desde então, que suas fábricas fiquem fechadas ou operem com baixa capacidade (Sohrabi et al., 2020).

Considerando que um dos maiores desafios enfrentados pelas organizações em momentos de crise é a dificuldade em tomar decisões pela falta de informações (Alves \& Lopes, 2019). Por se tratar de uma doença e de uma situação recente, as lacunas de conhecimento e informação ainda são cíclicas. Contudo, é essencial que as organizações adotem medidas para reduzir os impactos nocivos deste período de crise.

Durante mudanças rápidas e ameaças extremas, apenas dirigir o navio não é suficiente. Portanto, os líderes devem introduzir práticas organizacionais que aprimorem a capacidade da organização não apenas para suportar as incertezas dos tempos difíceis, mas também para emergir mais forte no futuro (Pereira, Budhwar, Temouri, Malik, \& Tarba, 2020).

A partir do aporte teórico é possível inferir que as medidas governamentais são imprescindíveis para auxiliar as organizações, singularmente, neste período de crise que afeta todos os segmentos de mercado. Assim, estratégias para minimizar tais impactos econômicos evitando o aumento expressivo do desemprego e da falência das organizações carecem de intervenções do Estado. À vista disso, elencou-se estratégias necessárias como: emergência nas ações relativas à saúde pública, regulamentação do teletrabalho, auxílio financeiro para as organizações, comunicação eficiente entre o Estado e organizações e a redução de impostos neste período de crise.

No que se refere aos ambientes digitais, estes configuram-se como mecanismos de interação e continuidade do trabalho à distância. Contudo, a aceleração das transformações digitais, investimento em infraestrutura de TIC, planejamento da força de trabalho, aprimoramento digital, investimento em marketing e branding e pesquisas do comportamento do consumidor pós-pandemia são componentes essenciais para as organizações somarem esforços frente à COVID-19.

A modalidade do teletrabalho se intensificou no contexto da pandemia, todavia é importante ter alguns cuidados e delinear estratégias para esta tendência de atividades laborais à distância. Subentende-se que a elaboração de políticas empáticas e responsivas, a manutenção da continuidade do trabalho, fornecimento e suporte para o trabalho, estabelecimento de política para o teletrabalho a longo prazo, orientação do tempo e a redução da jornada de trabalho são aspectos que devem ser implementados a fim de criar um ambiente favorável ao desenvolvimento das atividades.
A atenção à saúde mental é de extrema relevância, especialmente em um panorama com impactos imensuráveis em diversos aspectos. Diante disso, por meio destas medidas como a adoção de medidas e apoio ao bem-estar dos colaboradores, a criação de canais de acolhimento psicológico e a efetiva comunicação corporativa é possível fazer intervenções com o intuito de minimizar impactos negativos e promover saúde mental dos trabalhadores. A Tabela 1 sintetiza proposições que tendem a auxiliar as organizações a reduzirem os impactos da COVID-19.

Tabela 1

Proposições para auxiliar as organizações no contexto da pandemia

\begin{tabular}{|c|c|}
\hline Categorias & Proposições \\
\hline $\begin{array}{l}\text { Medidas } \\
\text { Governa- } \\
\text { mentais }\end{array}$ & $\begin{array}{c}\text { Emergência nas ações relativas à saúde pública; } \\
\text { Regulamentação do teletrabalho; } \\
\text { Auxílio imediato para micro, pequenas, médias e grandes } \\
\text { empresas; } \\
\text { Comunicação eficiente e efetiva; } \\
\text { Redução de impostos. }\end{array}$ \\
\hline $\begin{array}{c}\text { Ambientes } \\
\text { Digitais }\end{array}$ & $\begin{array}{c}\text { Aceleração das transformações digitais; } \\
\text { Minimização das lacunas na infraestrutura de TI; } \\
\text { Planejamento da força de trabalho; } \\
\text { Aprimoramento digital; } \\
\text { Investimento de marketing e branding; } \\
\text { Estudo do comportamento do consumidor pós-pandemia. }\end{array}$ \\
\hline Teletrabalho & $\begin{array}{l}\text { Elaboração de políticas empáticas e responsivas; } \\
\text { Manutenção da continuidade do trabalho; } \\
\text { Fornecimento de recursos e suporte necessários para o traba- } \\
\text { lho; } \\
\text { Estabelecimento de políticas para o trabalho remoto a longo } \\
\text { prazo; } \\
\text { Organização do tempo; } \\
\text { Redução da jornada de trabalho. }\end{array}$ \\
\hline $\begin{array}{l}\text { Saúde } \\
\text { mental }\end{array}$ & $\begin{array}{l}\text { Adoção de medidas e apoio ao bem-estar físico e emocional } \\
\text { dos colaboradores no trabalho ou em casa; } \\
\text { Criação de canais de acolhimento psicológico; } \\
\text { Comunicação corporativa frequente. }\end{array}$ \\
\hline
\end{tabular}

Nota. Fonte: elaborado pelas autoras.

A tentativa de propor algumas ações para enfrentamento da crise tem o intuito de minimizar os impactos sofridos pela pandemia. No entanto, é primordial elaborar um plano estratégico que visualize ameaças, riscos e possíveis cenários futuros, implicando em ações efetivas diante de situações inesperadas. Por outro lado, é necessário salientar as precauções para o público interno em manter boas práticas de higiene e saúde, recomendados pela Organização Mundial da Saúde, promover um ambiente de trabalho seguro e pensar em diligências pós-pandemia.

\section{Referências}

Aderaldo, I. L., Aderaldo, C. V. L., \& Lima, A. C. (2017). Aspectos críticos do teletrabalho em uma companhia multinacional. Cad. EBAPE.BR, 15, 511 533. https://doi.org/10.1590/1679-395160287

Alves, F., \& Lopes, L. (2019) Preparação para crises, uma vantagem competitiva: aprendendo com 4.500 casos. Pesquisa Global de Crises PwC 2019. Recuperado de: https://www.pwc.com.br/pt/estudos/servicos/consultorianegocios/2019/global-crisis-survev.html

American Psychiatric Association (2014). Manual diagnóstico e estatístico de transtornos mentais: DSM-5. Porto Alegre, RS: Artmed.

Asmundson, G. J. G., \& Taylor, S. (2020). How health anxiety influences responses to viral outbreaks like COVID-19: What all decisionmakers, health authorities, and health care professionals need to know. Journal of Anxiety Disorders, 71(102211). https://doi.org/10.1016/j. janxdis.2020.102211

Barros-Delben, P., Cruz, R. M., Trevisan, K. R. R., Gai, M. J. P., Carvalho, R. V. C, Carlotto, P. A. C . . Malloy-Diniz, L. F. (2020). Saúde mental em situação de emergência: Covid-19. Manuscrito submetido para publicação.

Bedford, J., Enria, D., Giesecke, J., Heymann, D. L., Ihekweazu, C., Kobinger, G., ... \& Ungchusak, K. (2020). COVID-19: towards controlling of a pandemic. The Lancet, 395 (10229), 1015-1018. http://doi.org/10.1016/S01406736(20)30673-5 
Behera, R. K., Gunasekaran, A., Gupta, S., Kamboj, S., \& Bala, P. K. (2020) Personalized digital marketing recommender engine. Journal of Retailing and Consumer Services, 53(101179). https://doi.org/10.1016/i. jretconser.2019.03.026

Brooks, S. K., Webster, R. K., Smith, L. E., Woodland, L., Wessely, S., Greenberg, N., \& Rubin, G. J. (2020). The psychological impact of quarantine and how to reduce it: Rapid review of the evidence. The Lancet, 395(10227), 912-920. https://doi.org/10.1016/S0140-6736(20)30460-8

Carr, B. G., \& Hollander, J. E. (2020) Virtually Perfect? Telemedicine for COVID-19. The New England Journal of Medicine. https://doi.org/10.1056/ NEJMp2003539

Chaves Júnior, J. E. R. (2017). O Direito do Trabalho e as plataformas eletrônicas. Em R. S. Melo \& C. J. Rocha (Coord.), Constitucionalismo, trabalho, seguridade social e as reformas trabalhista e previdenciária (pp. 357-366). São Paulo: LTr.

Chen, Q., Liang, M., Li, Y., Guo, J., Fei, D., Wang, L., ... \& Zhang, Z. (2020). Mental health care for medical staff in China during the COVID-19 outbreak. The Lancet Psychiatry, 7(4), 15-16. https://doi.org/10.1016/S22150366(20)30078-X

Chui, C. H. K., \& Chan, C. H. (2019) The role of technology in reconfiguring volunteer management in nonprofits in Hong Kong: Benefits and discontents. Nonprofit Management and Leadership, 30(1), 89-111. https://doi. org/10/1002/nml.21369

Croda, J., Oliveira, W. K. D., Frutuoso, R. L., Mandetta, L. H., Baia-da-Silva, D. C., Brito-Sousa, J. D., ... \& Lacerda, M. V. G. (2020). COVID-19 in Brazil: advantages of a socialized unified health system and preparation to contain cases. Revista da Sociedade Brasileira de Medicina Tropical, 53. https://doi. org/10.1590/0037-8682-0167-2020

Cruz, R. M., Borges-Andrade, J. E., Moscon, D. C. B., Micheletto, M. R. D., Esteves, G. G. L., Delben, P. B., ... \& Carlotto, P. A. C. (2020). COVID-19; emergência e impactos na saúde e no trabalho. Revista Psicologia Organizações e Trabalho, 20(2), I-III. https:// doi.org/10.17652/rpot/2020.2.editorial

Decreto $n^{\circ} 10.211$, de 30 de janeiro de 2020. Dispõe sobre o Grupo Executivo Interministerial de Emergência em Saúde Pública de Importância Nacional e Internacional - GEI-ESPII. Brasília, DF. http://www.planalto.gov.br/ ccivil 03/ato2019-2022/2020/decreto/D10211.htm

Duan, L., \& Zhu, G. (2020). Psychological interventions for people affected by the COVID-19 epidemic. The Lancet Psychiatry, 7(4), 300-302. https://doi. org/10.1016/S2215-0366(20)30073-0

Felstiner, A. (2011) Working the Crowd: Employment and Labor Law in the Crowdsourcing Industry. Berkeley Journal of Employment and Labor Law, 32(1). https://wtf.tw/ref/felstiner.pdf

Filardi, F., Castro, R. M. P., \& Zanini, M. T. F. (2020) Vantagens e desvantagens do teletrabalho na administração pública: análise das experiências do Serpro e da Receita Federal. Cad. EBAPE.BR, Rio de Janeiro, 18(1), 28-46. https:// doi.org/10.1590/1679-395174605

Gatera, G., \& Pavarini, G. (2020) COVID-19: what is next for public health? The lancet, 395, 542-545. https://doi.org/10.1016/S0140-6736(20)30374-3

Hellewell, J., Abbott, S., Gimma, A., Bosse, N. I., Jarvis, C. I., Russell, T. W., .. \& Flasche, S. (2020). Feasibility of controlling COVID-19 outbreaks by isolation of cases and contacts. The Lancet Global Health, 8, 488-496. https:// doi.org/10.1016/S2214-109X(20)30074-7

Huang, C., Wang, Y., Li, X., Ren, L., Zhao, J., Hu, Y., \& Cheng, Z. (2020) Clinical features of patients infected with 2019 novel coronavirus in Wuhan, China. The Lancet, 395(10223), 497-506. https://doi.org/10.1016/S01406736(20)30183-5

Kandel, N., Chungong, S., Omaar, A., \& Xing, J. (2020). Health security capacities in the context of COVID-19 outbreak: an analysis of International Health Regulations annual report data from 182 countries. The Lancet, 395(10229), 1047-1053. https://doi.org/10.1016/S0140-6736(20)30553-5

Medida Provisória $n^{\circ}$ 927, de 22 de março de 2020. Dispõe sobre as medidas trabalhistas para enfrentamento do estado de calamidade pública pelo Decreto Legislativo $\mathrm{n}^{\circ}$ 6, de 20 de março de 2020. Brasília, DF. http:// www.in.gov.br/en/web/dou/-/medida-provisoria-n-927-de-22-de-marcode-2020-249098775

Medida Provisória n ${ }^{\circ}$ 936, de 1 de abril de 2020. Institui o Programa Emergencial de Manutenção do Emprego e da Renda e dispõe sobre medidas trabalhistas complementares para enfrentamento do estado de calamidade pública reconhecido pelo Decreto Legislativo n 6, de 20 de março de 2020. Brasília, DF. http://www.in.gov.br/en/web/dou/-/medida-provisoria-n-936-de-1de-abril-de-2020-250711934

Medida Provisória $n^{\circ}$ 944, de 3 de abril de 2020. Institui o Programa Emergencial de Suporte a Empregos. Brasília, DF. http://www.in.gov.br/en/web/dou/-/ medida-provisoria-n-944-de-3-de-abril-de-2020-251138829

Messenger, J. C. (2019) Telework in the 21st century: an evolutionary perspective. Cheltenham, UK: Edward Elgar Publishing. https://doi. org/10.4337/9781789903751

Parodi, S. M., \& Liu, V. X. (2020). From Containment to Mitigation of COVID-19 in the US. American Medical Association, 323(15), 1441-1442. https://doi.org/10.1001/jama.2020.3882
Pereira, V., Budhwar, P., Temouri, Y., Malik, A., \& Tarba, S. (2020) Investigating investments in agility strategies in overcoming the global financial crisis - The case of Indian IT/ BPO offshoring firms. Journal of International Management, 100738. https://doi.org/10.1016/j.intman.2020.100738

Portaria $n^{\circ} 188$, de 3 de fevereiro de 2020. Declara Emergência em Saúde Pública de importância Nacional (ESPIN) em decorrência da Infecção Humana pelo novo Coronavírus (2019-nCoV). Brasília, DF. http://www.in.gov.br/web/ dou/-/portaria-n-188-de-3-de-fevereiro-de-2020-241408388

Resolução CGSN n 154 , de 3 de abril de 2020. Dispõe sobre a prorrogação de prazos de pagamento de tributos no âmbito do Simples Nacional, em razão da pandemia da Covid-19. Brasília, DF. Recuperado de http:// normas.receita. fazenda.gov.br/sijut2consulta/link.action?visao=anotado\&idAto $=108368$

Rindfleisch, A. (2019) The Second Digital Revolution. Marketing Letters, p. 1-5.

Sohrabi, C., Alsafi, Z., O’Neill, N., Khan, M., Kerwan, A., Al-Jabir, A., ... \& Agha, R. (2020). World Health Organization declares global emergency: A review of the 2019 novel coronavirus (COVID-19). International Journal of Surgery, 76, 71-76. https://doi.org/10.1016/i.ijsu.2020.02.034

Silva, M. F. G. (2020). Uma história sobre pandemia (Covid-19), isolamento e fundamentos microeconômicos de políticas públicas. Cadernos Gestão Pública e Cidadania, 25(80), 1-11. https://doi.org/10.12660/cgpc.v25n80.81290

Ting, D. S. W., Carin, L., Dzau, V., \& Wong, T. Y. (2020). Digital technology and COVID-19. Nature Medicine, 26, 458-464. https://doi.org/10.1038/s41591020-0824-5

Veiga, A. G. B., Oliveira, L. C. D., \& Mendes, R. A. O. (2020). A viabilidade do teletrabalho na administração pública brasileira. Brazilian Journal of Development, 6(3), 12745-12759. https://doi.org/10.34117/bjdv6n3-222

Wang, C. J., Ng, C. Y., \& Brook, R. H. (2020). Response to COVID-19 in Taiwan: big data analytics, new technology, and proactive testing. Jama, 323(14), 1341-1342. https://doi.org/10.1001/jama.2020.3151

Wilder-Smith, A., Chiew, C. J., \& Lee, V. J. (2020). Can we contain the COVID-19 outbreak with the same measures as for SARS?. The Lancet Infectious Diseases, 20, e102-107. https://doi.org/10.1016/S1473-3099(20)30129-8

Wong, J. E., Leo, Y. S., \& Tan, C. C. (2020). COVID-19 in Singapore- current experience: critical global issues that require attention and action. Jama, 323(13), 1243-1244. https://doi.org/10.1001/jama.2020.2467

Wu, W., Liang, Z., Zhang, Q., \& Zhang, H. (2020) Coupling relationships and synergistic mechanisms between technology management capability and technological capability in product innovation: a simulation study. Technology Analysis \& Strategic Management, 1-15. https://doi.org/10.1080/09537325.2 $\underline{020.1743261}$

Xiao, Y., \& Torok, M. E. (2020) Taking the right measures to control COVID-19. The Lancet, 20(5), 523-524. https://doi.org/10.1016/ S1473-3099(20)30152$\underline{3}$

Yaseen, S. G., \& Al Omoush, K. S. (2020). Mobile Crowdsourcing Technology Acceptance and Engagement in Crisis Management: The Case of Syrian Refugees. International Journal of Technology and Human Interaction, 16(3), 1-23. https://doi.org/10.4018/IJTHI.2020070101

\section{Informações sobre as autoras:}

\section{Beatriz Leite Gustmann de Castro}

Av. Roraima no 1000 Cidade Universitária

Bairro Camobi - Santa Maria/RS

CEP: $97105-900$

E-mail: beatriz_gustmann@hotmail.com

\section{Joice Beatriz Bock de Oliveira}

E-mail: joicebdeoliveira@gmail.com

\section{Lísia Quoos Morais}

E-mail: lisiaquoosmorais@gmail.com

Maria Julia Pegoraro Gai

E-mail: mariajuliapgai@gmail.com 